\title{
EVALUACIÓN DEL PERFIL DE EGRESO Y PRÁCTICAS PROFESIONALES EN ESTUDIANTES DE PEDAGOGÍA EN EDUCACIÓN BÁSICA, EN LA UNIVERSIDAD CATÓLICA SILVA HENRÍQUEZ (UCSH), 2009
}

\section{Dra. Susana Barrera A*}

\section{RESUMEN}

Esta investigación se propone diagnosticar el logro de competencias del perfil de egreso alcanzado por estudiantes, y las dificultades en sus prácticas pedagógicas, en la carrera de Pedagogía en Educación Básica, con referencia en los Planes Comunes de Universidad (PCU) y de Educación (PCEDUC), y las áreas de formación generalista. Utiliza tests, con ítems problematizadores donde el evaluado aplica habilidades formadas. Los resultados corresponden a 66 voluntarios, de 98 egresados. En PCEDUC y Ciencias Naturales más de la mitad de egresados alcanzan logros regularmente suficientes; en PCU, Matemática, Ciencias Sociales y Educación Artística se concentran logros insuficientes, mientras que en Lenguaje un tercio evidencia logros suficientes. No hay diferencia significativa en los resultados del Programa por jornadas. En las prácticas se destacan problemas en la supervisión, manejo de estrategias de enseñanza y de convivencia.

Palabras clave: Evaluación de logros, perfil de egreso, formación docente, práctica profesional

\section{GRADUATE PROFILE AND PROFESSIONAL PRACTICE IN TEACHINGEVALUATIONOFSTUDENTSOFELEMENTARY TEACHING EDUCATION AT UNIVERSIDAD CATÓLICA SILVA HENRÍQUEZ (UCSH), 2009}

\begin{abstract}
The purpose of this research is to diagnose the achievement of the competences of the graduate profile achieved by students and the difficulties in their internships regarding the Program of Education in Elementary Education, with reference to the Common Plan of University (PCU) and Common Plan of Education (PCEDUC), and the areas of general training. It uses tests, with items in context where he applies the skills acquired. Results correspond to 66 volunteers of 98 graduates. In the Common Plan Education and Natural Sciences for more than half of graduates achieve regularly sufficient progress, in Common Plan University, Mathematics, Social Science and Art focus on insufficient achievements, while one third show enough achievements on Language. There is no statistically significant difference in results by the type of Program. In the internships, stand out problems with supervision, the managing strategies, and the school coexistence.
\end{abstract}

Key Words: Evaluation of achievements, graduate profile, initial teacher training, internships.

* La experiencia es parte del Proyecto institucional original Una experiencia piloto en la UCSH: Evaluación del perfil de egreso en programas de pedagogía, iniciado a mediados de 2008, en el que la autora fue la Investigadora Responsable. Para su financiamiento siguió procedimientos regulados por la Dirección de Investigación y Postgrado de la UCSH. 


\section{Introducción}

La evaluación de los egresados de programas de formación inicial docente comienza a tener realce, a partir de la promulgación de la Ley 20.I29 de Aseguramiento de la Calidad de la Educación Superior (2006), como una práctica de la gestión educacional útil para la toma de decisiones de políticas institucionales. En ellas se regulan procesos de evaluación en egresados de carreras de pedagogía, en procura de detectar insuficiencias formativas, e introducir correcciones oportunas en la formación de profesores, y consecuentemente, obtener mejores desempeños expresados en aprendizajes de los estudiantes.

\section{Instalación de las prácticas evaluativas docentes}

En Chile, desde los años noventa, con el advenimiento de la democracia al país, se han desarrollado sistemáticamente experiencias de reformas orientadas a mejorar la educación formal en todos sus componentes, siendo uno de ellos la formación de profesores. El proyecto de Fortalecimiento de la Formación Inicial Docente, realizado por el Ministerio de Educación (Mineduc), fue uno de ellos, marcando un hito importante en la intervención de políticas públicas a favor del mejoramiento de la calidad de la formación docente. La transformación curricular, la renovación metodológica y la dinamización de procesos reflexivos y comprensivos entre otros cambios, fueron logros significativos (Ávalos, 2002). Por ese entonces se gestaron las bases del Marco para la Buena Enseñanza (MBE) que posteriormente se transforma en el principal referente para las instituciones formadoras de profesores, vigente a la fecha (Mineduc, 2003).

Con la Ley I9.7I5 (200I) se crea la Asignación de Excelencia Pedagógica (AEP), que pone en práctica un mecanismo de evaluación voluntaria de profesores en ejercicio, en el que se premia el mérito académico y se favorece la permanencia de funciones del docente vinculado al sistema público y subvencionado, otorgándole incentivos económicos a quienes demuestran conocimientos y desempeños 
satisfactorios, bajo el parámetro del MBE, experiencia similar a la de Estados Unidos, a través del National Board for Professional Teaching Standards (NBPTS).

A contrapunto de la AEP, por la misma época, se evalúa a una cohorte de estudiantes de carreras pedagógicas al momento de ingresar y egresar de la formación docente, utilizando la prueba Praxis I elaborada por el Educational Testing Service. Una de las conclusiones del estudio revela que los evaluados demuestran limitadas habilidades básicas en matemáticas y lenguaje, atribuyendo esto al legado de la educación secundaria, sin que su paso por la universidad pudiera haber contribuido a una mejora (Larrondo, 2001, 2007). Esta experiencia levantó una advertencia, que posteriormente sumó argumentos para avanzar en la evaluación de docentes noveles sobre el desarrollo de competencias adquiridas en el proceso formador y proyectar medidas de mejora en coordinación con las instituciones formadoras.

Estudios posteriores que relacionan resultados del Simce con datos del Programa AEP evidencian que profesores competentes o destacados durante los tres años previos a rendir la prueba claramente obtuvieron mejores resultados que sus compañeros de generación que no babian tenido un bistorial de profesores bien evaluados (Mineduc, 2008a, p. I5).

\section{Evidencias internacionales}

Este hallazgo se sustenta teóricamente en estudios precedentes que demustran que, más que el número de estudiantes por clase, el principal impulsor de las variaciones en el aprendizaje escolar es la calidad de los docentes (Barber \& Mourshed, 2008, p. I2), con énfasis en sistemas educativos asiáticos y europeos. Similares afirmaciones son confirmadas en el contexto norteamericano (Millman \& Darling-Hammond, I997; Darling-Hammond, 2000, Darling-Hammond \& McLaughlin, 2004), sobre el cual existe suficiente evidencia empírica para afirmar que la formación de profesores pudiera ser un buen predictor del aprendizaje de los estudiantes. 


\section{Aportes comprensivos y factores asociados}

Sin embargo, en el contexto chileno un trabajo reciente (Araya, Santelices, Taut, Manzi \& Miño, 20I0) aporta nuevas luces sobre el programa AEP; a la letra señala que las consecuencias que se pretende que favorezca el programa quedan expresadas como un conjunto de supuestos y creencias, en lugar de un modelo causal con nexos claros entre insumos, actividades, resultados inmediatos y metas finales (p. I I), quedando pendiente, según los autores, una próxima etapa de investigación en la que se busca aportar más evidencia empírica sobre los efectos observados en dicho Programa, ya sean esperados o inesperados.

En medio de la difusión, el realce político e inclusive equivocadamente publicitario que se hace a los resultados de evaluaciones de profesores en servicio y estudiantes en formación docente, pasan desapercibidos o poco difundidos y desconocidas las constataciones que hacen las investigaciones del Laboratorio Latinoamericano de Evaluación de la Calidad (UNESCO, 2000, 2008), en el sentido de que la escuela en su conjunto hace la diferencia en tanto tiene un peso significativo en su contribución al aprendizaje del estudiante, y dentro de ésta se destaca la relevancia del clima escolar expresado en un ambiente positivo de respeto y acogida que predispone al aprendizaje. Un análisis complementario de los resultados académicos de los escolares relacionados con el coeficiente Gini demuestra que la desigualdad del ingreso está relacionada de manera inversa con el aprendizaje (UNESCO, 2008, p. 52), todas variables de las que se habla poco.

En ese mismo sentido Abadzi (2008), sin desconocer las condiciones de pobreza y de desventaja de los estudiantes, destaca que el manejo del currículo, las prácticas evaluativas diagnósticas, y el manejo de textos que hace el docente en clases pudieran estar influyendo positivamente en los estudiantes.

Evaluación de egresados de pedagogía

Los estudios de CINDA (2004) también aportan a la evaluación de competencias en egresados de universidades en la perspectiva de 
la acreditación profesional. En el caso de las pedagogías diferencia las competencias de egreso de las profesionales, siendo la primeras la bases de éstas para el desempeño en el aula, atribuyéndole a la reflexión de la práctica una importancia clave (Schön, 2002) para que el docente genere respuestas contextualizadas y creativas, lejos de caer en rutinas tradicionales.

La integración de Chile a la OCDE en 2009 ha significado una revisión y mejora en el conjunto de sus políticas públicas con fuertes tensionamientos políticos provenientes de informes que evalúan y formulan recomendaciones en diversas áreas de desarrollo nacional que comprometen mejoras. En sucesivos informes, en materia de educación superior se valoran los avances en acreditación institucional, pero sugieren profundizar en equidad y en la implementación de sistemas de aseguramiento de la calidad. También plantean la importancia de certificar a profesionales de la salud y de la educación para su ejercicio. En el último reporte destaca mejorar el currículo de formación, incentivar a estudiantes de buen rendimiento para que estudien pedagogía, y reiteran la aplicación de evaluaciones a los egresados de pedagogía (OCDE, 2010, p. 7I).

Asumiendo estas preocupaciones el Consejo Superior de Educación (CSE, 20I0) abrió el debate sobre la calidad de los egresados pudiendo apreciarse pocas y muy diversas experiencias de evaluación, casi todas autorreferidas al modelo de formación institucional, quedando en evidencia lo nuevo de la temática y el desafío de crear, probar y desarrollar metodologías válidas en este campo.

La gradual implementación de la Ley 20.129 de Aseguramiento de la Calidad de la Educación Superior (2006) en la Estrategia Bicentenario para la Formación Docente (Mineduc, 2008b), proyecta la puesta en marcha de procesos evaluativos a los egresados, comenzando por Pedagogía en Educación Básica (PEB). De ese modo el Programa "Inicia" aplica la primera experiencia piloto con carácter diagnóstico, en la perspectiva de profundizar en la transformación curricular de la formación y las prácticas pedagógicas en las instituciones formadoras (Mineduc, 2009). 
Los resultados de la última evaluación (2010) han sido controversiales por la metodología utilizada, la baja representatividad de egresados que voluntariamente se presentaron por institución, que ponen en duda la veracidad y generalización de resultados, especialmente cuando el Mineduc publica por prensa un ranking de Universidades con datos poco cré́bles que causan impacto negativo en la sociedad y las instituciones superiores.

\section{Políticas institucionales en la UCSH}

En este contexto la UCSH, siguiendo las directrices de políticas nacionales, formula su Modelo de Formación (2003) en el que conceptualiza el perfil de egreso como perfil de formación dado por el

conjunto de conocimientos y babilidades, orientaciones y actitudes que se consideren necesarias para que un estudiante alcance el título profesional y $/ 0$ grado académico de su Programa de Formación, y que lo babilita para el desempeño de las actividades propias de su campo ocupacional (p. I4).

Por entonces el Modelo compromete un currículo orientado al desarrollo de competencias profesionales y disciplinares y de orden genérico, dadas por aquellas que comportan el desarrollo humano, ético, social y cultural de los estudiantes en formación. Determina el Plan Común con actividades curriculares que agrupan áreas formativas, identificándose el Plan Común de Universidad que incluye aquellas relacionadas con desarrollo personal y de identidad salesiana, y el Plan Común de Educación, con asignaturas propias de la formación pedagógica, investigativa, y de gestión escolar.

Este último, adopta el MBE para la formulación de 19 competencias (UCSH, 2006) que son ajustadas para las I0 actividades curriculares, con asignación de horas en créditos transferibles, como unidad de equivalencia de estudios. En 2008 la UCSH se ve fortalecida con la implementación de tres Programas de Mecesup 2: el diseño del Programa de Pedagogía en Ciencias Naturales, la cualificación de las prácticas profesionales docentes, e iniciativas innovadoras de 
apoyo a los estudiantes que ingresan con insuficientes competencias básicas.

Respecto de esto último, según datos frescos de la Dirección de Planificación de la UCSH indican que, si bien los puntajes de la PSU están alrededor y por encima de la media nacional y las notas en el entorno de 5,5, y un poco más, la debilidades con que llegan los estudiantes obliga a la institución a movilizar estrategias de mejoramiento de las habilidades instrumentales y sociales, ya que estas condiciones de entrada acompañan y dificultan el aprendizaje y logro del perfil de egreso. Tan relevante es esta situación que en el último informe de Autoevaluación Institucional, la universidad reconoce que son insuficientes los esfuerzos que hace para que los malos estudiantes concluyan su proceso formativo, y en consecuencia, es posible que el perfil de egreso se esté logrando en forma limitada en los estudiantes, en los diversos programas (UCSH, 2008, pp. 22-23).

Con referencia a los señalamientos a la PSU, lo señalado es coherente con estudios nacionales que demuestran la validez predictiva del puntaje de la PSU de Matemática y Ciencias, respecto del rendimiento de los estudiantes al finalizar el primer año de formación universitaria (CRUCH, 2008), hecho que inclusive la UCSH constata con datos propios (Barrera, 2010a, p. 53, 2010b, pp. 65-68). Sin embargo, sobre este tema hay mucho que profundizar porque recientes indagaciones remarcan el carácter selectivo y discriminador de la PSU, toda vez que su resultado es una prueba que sintetiza y legitima las desigualdades sociales en el país, observándose que los puntajes están asociados directamente a la distribución del ingreso. Tanto es así que los datos de Simce de 8vo año de primaria predicen los resultados de los estudiantes cuatro años después, cuando rinden la PSU (Contreras, Corvalán \& Redondo, 2007, p. 46).

La evaluación de los egresados de programas de pedagogía en la UCSH se sitúa dentro de esta misma lógica de preocupación por la calidad de la formación inicial docente. En los hechos, en 2008 con la finalidad de diagnosticar la situación de la formación de 
profesores, se diseñaron pruebas para evaluar los PCU y PCEDUC en los egresados de todas las carreras de pedagogía, y para PEB se diseñó un instrumento específico, pero debido a la falta de convocatoria, sólo fue posible ejecutar la evaluación al resto de las pedagogías por dos gestiones consecutivas (Barrera, 2009, 2010), y en el Programa de PEB recién aplicó instrumentos al año siguiente; en esta oportunidad se presenta una parte de los resultados encontrados.

En paralelo a la experiencia de cuño propio, se destaca que la UCSH ha participado en forma consecutiva con más de un $80 \%$ de estudiantes en las evaluaciones del Programa "Inicia", siendo una muestra de su voluntad para mejorar de manera continua la calidad de su oferta formativa docente. Se deja en claro que, por cuestiones de diseño metodológico, y tipo de competencias evaluadas, no es posible comparar los resultados del Proyecto "Inicia" 2009 con la evaluación realizada por la UCSH el mismo año; aun cuando se trata de la misma población, tanto el plan de evaluación desarrollado como el marco de referencia curricular son diferentes.

\section{Objetivo}

Relevar un diagnóstico sobre el logro alcanzado por los egresados del Programa de Pedagogía en Educación Básica, de la gestión 2009, de la Universidad Católica Silva Henríquez, respecto de: a) las competencias declaradas en el perfil de egreso, en referencia a los Planes Comunes de Universidad y de Educación y las áreas de formación generalista, y b) las dificultades encontradas en las prácticas profesionales.

\section{Metodología}

En la consecución de esta evaluación participaron cerca de treinta docentes de la universidad, ya como coordinadores, integrantes de grupos de consulta, diseñadores de ítems o en juicios de expertos, protagonismo que suma a la transparencia, compromiso e instalación de estos procesos, en las estrategias de aseguramiento de la calidad. 
La población evaluada fue de 66 estudiantes de los 98 a punto de egresar en 2009 que se presentaron voluntariamente; esto representa el $67 \%$ del total, con las desagregaciones por jornada diurna y vespertina, esta última con la totalidad de sus egresados.

Los instrumentos aplicados fueron dos en formato de cuadernillos. El primero evalúa los Planes Comunes. Se utilizaron dos grupos de ítems que se organizaron en dos formas de pruebas estructuradas como bloques cruzados. Cada cuadernillo comprendió una subprueba de PCU con 34 ítems y otra de PCEDUC con 49 ítems.

La prueba generalista que refiere a las áreas curriculares formativas del profesor de $\mathrm{PEB}$, comprendió también cinco grupos de ítems identificados como subpruebas, organizadas en dos formas de bloques cruzados que hicieron un total de 97 ítems desagregados por Lenguaje, Matemática, Ciencias Naturales, Ciencias Sociales y Educación Artística, con 37, 26, I I, I2 y II ítems, respectivamente. Cada subprueba contó con un diseño de tabla de especificaciones, bajo dos criterios matriciales:

- dimensiones del saber, en relación a conceptos, procedimientos y actitudes y valores, que se expresan en competencias del saber conocer, saber hacer y ser/convivir, respectivamente; dentro de cada una de ellas se distribuyeron las competencias seleccionadas según la relevancia atribuida por los académicos consultados por los coordinaciones de equipos de trabajo por cada Plan y PEB, y - niveles de pensamiento deductivo, crítico y aplicado.

Los ítems fueron sometidos a Juicio de expertos y valorados estadísticamente para estimar su confiabilidad. Las subpruebas de los Planes Comunes, Lenguaje y Matemática se estima que contaban con suficientes ítems para tener un buena cobertura evaluativa por cada competencia, demostrando valores psicométricos adecuados; los ítems de Ciencias Naturales, Ciencias Sociales y Educación Artística demostraron también buenos valores métricos, sin embargo se estima que los resultados deben ser tomados como hipótesis a retomar en nuevos estudios con tamaños más grandes de población. 
Tratándose de varios grupos de ítems asumidos como subpruebas, se procedió a un análisis de la dimensionalidad del instrumento. Para tal efecto se usó un procedimiento de análisis factorial, de componentes principales basados en los residuos de Rasch, que proporciona información útil para determinar si el instrumento es unidimensional o no, lo cual se fundamenta en la varianza de los datos dada por la dimensión específica de los constructos evaluados. Los datos constatan que se están midiendo varias dimensiones, por lo que correspondió procesar cada subprueba por separado.

Con esta información se procedió a la construcción de una escala de puntaje que proporciona el Modelo de Rascb con el programa estadístico Winstep. Utiliza escala logit con media cero y desviación estándar de $\underline{I}$. Como presenta valores negativos y positivos, de poca comprensión a primera vista, los resultados se transforman a una escala que va de 0 a 100 puntos, de más fácil manejo, con todos los valores positivos. Esta escala se utiliza con frecuencia en evaluaciones de carácter educativo y es comprensible ya que tiene promedio de 50 puntos y una desviación estándar de 10 puntos.

En el Modelo Rasch se identifican puntos de anclaje que representan parámetros de dificultad de los ítems. La escala de habilidad que resulta por cada subprueba, determina los puntos de anclaje para cada nivel marcando los intervalos de clase por cada nivel de logro.

\section{Conceptualización de los niveles de logro}

El Cuadro I describe cada nivel de logro del estudiante evaluado, al término de su proceso formativo. Si bien los ítems de las pruebas lo posicionan frente a escenarios posibles, el trasfondo que se observa es la probabilidad de su desempeño futuro, en función de su quehacer pedagógico y la capacidad para aplicar y movilizar lo que sabe.

El procesamiento de la prueba y las subpruebas permitió encontrar diferentes grados de dificultad en la resolución de problemas. De ese modo, la información cuantitativa expresada en puntos de 
anclaje determina el valor promedio de las dificultades que tiene cada estudiante al momento de responder. En otras palabras, la probabilidad que tiene de responder correcta o incorrectamente, dependerá de su habilidad para resolver la situación dada.

\section{Cuadro Único}

UCSH, 2009: Programa de Pedagogía en Educación Básica. Concepto evaluativo y descripción de los niveles de logros según los puntajes de habilidad de la prueba porárea dis ciplinar

\begin{tabular}{|c|c|c|}
\hline $\begin{array}{l}\text { Denominacion } \\
\text { literal por nivel }\end{array}$ & Concepto & Descripcion del nivel \\
\hline & $\begin{array}{c}\text { Muy } \\
\text { insuficiente }\end{array}$ & 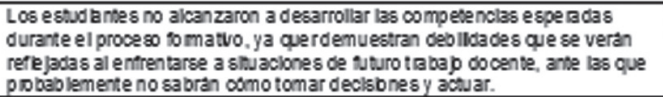 \\
\hline & Insuficiente & 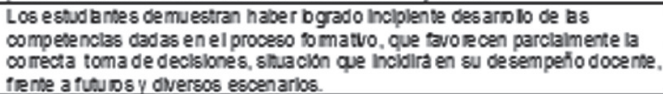 \\
\hline & $\begin{array}{c}\text { Regulo rm ente } \\
\text { s uficiente }\end{array}$ & 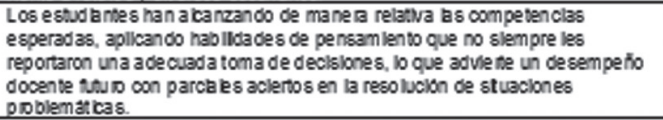 \\
\hline & Suficiente & $\begin{array}{l}\text { Los estuda ntes han desarrollado de manera esperada las competenclas } \\
\text { evaluadas, movilzando adecuad os procesos de pensam lento en la toma de } \\
\text { declib nes, por tanto, b mas posilole es que al momento de ejercer la funcion } \\
\text { docente b hacan enfrentando satista ctorlamente la Resolucion de prob emas. }\end{array}$ \\
\hline
\end{tabular}

Sistematización de la información cualitativa

En la prueba institucional se introdujo una sección con tres ítems abiertos con doble propósito: uno, evaluar producción escrita y otro, conocer las dificultades que los evaluados encuentran en su práctica profesional docente. En este informe sólo se presentan resultados de esta última. El procedimiento general seguido con los 253 registros textuales fue el siguiente:

- Las tres hojas de la prueba conteniendo textos de puño y letra de los estudiantes fueron separadas del cuadernillo de la prueba institucional manteniendo un código identificador correspondiente al evaluado, luego digitalizadas en planilla Excel, haciendo las correcciones ortográficas a efectos de edición, sin alterar la redacción ni las expresiones idiomáticas.

- Del primer ítem sobre la identificación de las dificultades propiamente se recogieron hasta seis frases pero considerando lo abundante de los textos se optó por trabajar con los tres 
primeros textos porque el resto mantenía ciertas constantes de respuestas.

- En cada texto se identificó la idea principal y se agrupó por temática asociada a un mismo problema a esta agrupación se le denominó categoría interpretativa. Surgieron I0 categorías, ocho de las cuales se confirmaron de la experiencia piloto de 2009, emergiendo dos nuevas: Necesidades especiales y Contexto y riesgo social, con importantes testimonios que la ilustraban.

- Luego se denominaron, en orden de aparición de los textos escritos: Ira respuesta, 2da respuesta y 3 ra respuesta y se codificaron con un número las respuestas por categoría de problema, conformando de este modo una base de datos cuantitativa sin perder los textos, que se pasó a SPSS a fin de generar tablas y gráficas.

- Al analizar el contenido de los textos agrupados por respuesta, surgieron expresiones clave que se denominaron descriptores de la categoría.

- De cada descriptor se seleccionaron algunos testimonios o imágenes verbales más llamativas y representativas de problema o dificultad encontrada. De este modo las imágenes verbales de varios descriptores conformaron los elementos argumentativos que dieron pie a un momento interpretativo de la situación dada tratando de establecer relaciones con otros.

\section{Resultados}

El perfil de los evaluados indica que de los 76 estudiantes la gran mayoría corresponde a población femenina (96\%), casi la mitad egresa en 2009 cumpliendo 23 años o menos, y un tercio lo constituye población de adultos jóvenes de 26 años o más. La mayoría son solteros, la mitad declara haber trabajado en el área de educación mientras cursaba estudios superiores y, a excepción de muy pocos, la mayoría concluye su formación sin haber discontinuado sus estudios. 
Los antecedentes académicos de los evaluados disponibles marcan dos momentos: antes de ingresar a la universidad, vistos desde los indicadores producto de la educación media, además de los puntajes de la prueba de selección universitaria, y después de concluido su proceso formativo universitario, a través del promedio de notas del Plan de Estudio.

Los datos indican que:

- Más de la mitad de los evaluados (69\%) estuvo en colegios particulares subvencionados, y en segundo lugar en colegios de dependencia municipal (23\%).

- El área de enseñanza de formación de procedencia, es mayoritariamente científico-humanista (77\%), y en menor proporción técnico profesional (21\%).

- El promedio de notas de enseñanza media comporta un $60 \%$ de estudiantes con promedio igual o mayor a 5,5 en escala de I a 7 .

- El puntaje de ingreso en PSU indica que un $44 \%$ de estudiantes ingresó con promedio de Lenguaje y Matemática igual o menor a 449,8 puntos; alrededor de un $20 \%$ lo hizo con puntajes entre 450 y 499,9 puntos, y el resto con puntajes mayores a éstos, siendo muy pocos los estudiantes que se sitúan en el rango de 600 puntos o más.

\section{Prueba generalista ${ }^{\mathrm{I}}$}

Rendimiento en puntaje

Estos resultados se expresan en puntajes promedios en escala estandarizada de 0 a 100 puntos. Está incluidos todos los ítems considerados efectivos por cada una de las subpruebas

I Barrera, S. (2010b). Informe técnico. Experiencia piloto evaluación del perfil de egress, Programa de Pedagogía en Educación Básica, gestión 2009. Santiago de Chile: Dirección de Docencia de Pregrado, UCSH, pp. 24 a 34 y Anexo 3, pp. 75 a 79. Información disponible para consultar detalles de Tablas de Especificaciones. 
correspondientes a las áreas disciplinares evaluadas, diferenciados por tipo de jornada en que la universidad imparte PEB. Los promedios son bastante similares con leves variaciones y se mueven muy cercanos a la media de 50 puntos (Gráfica I).

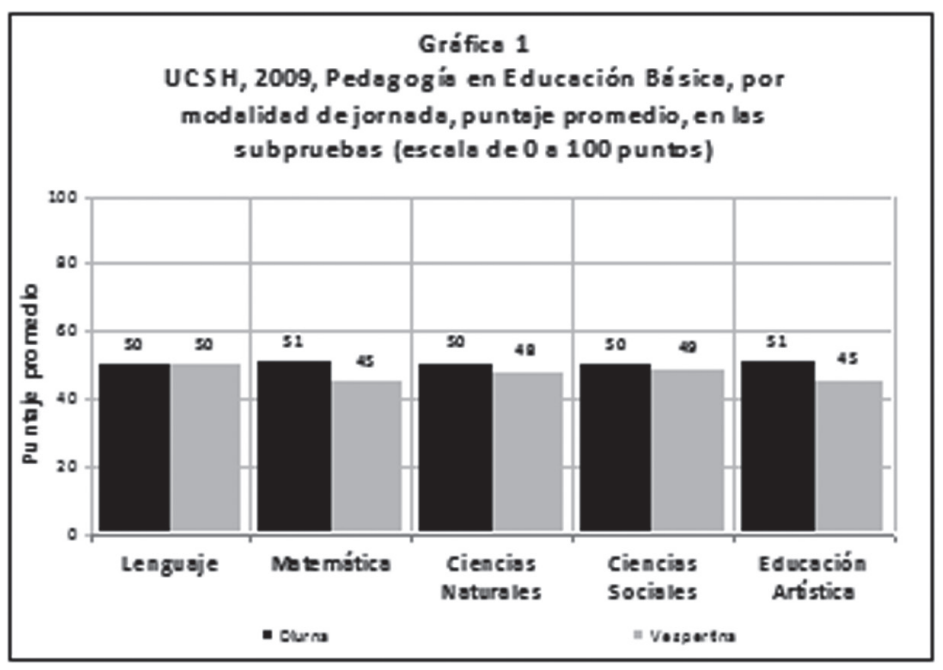

Los puntajes promedio y demás valores estadísticos por subprueba por cada jornada son muy similares, con una mayor dispersión de la población en los estudiantes de jornada vespertina, respecto de la diurna. No obstante, una vez aplicado el test $t$ de Student con el refinamiento de Welch para varianzas posiblemente no iguales, en estudiantes de una y otra jornada se constata que no existen diferencias significativas, lo cual indica que ambas poblaciones tienen un comportamiento similar en los resultados.

Niveles de logros en porcentaje de población

De manera global el área de Lenguaje es la que tiene mejor comportamiento con un 30\% de estudiantes con Suficiente (30\%) y la mitad con logros Regularmente suficientes. Esto último también se observa en Ciencias Naturales, a diferencia de Matemática con un $8 \%$ de estudiantes con logro Suficiente y la mitad de estudiantes con logro Muy insuficiente. Sociales y Educación Artísticas predominan 
logros Insuficientes, inclusive en esta última un tercio de estudiantes con logro Muy insuficiente (Gráfica 2).

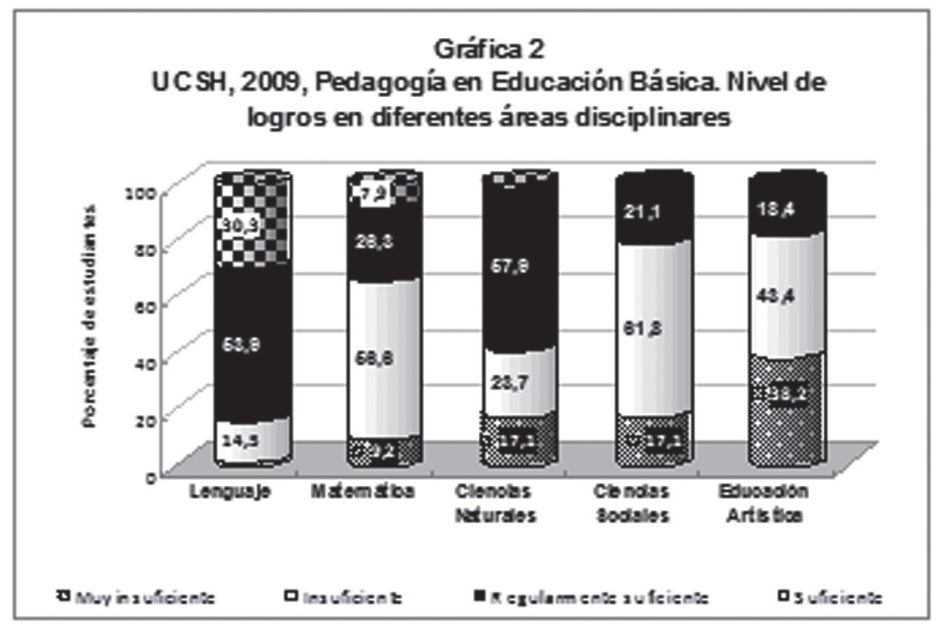

Dimensiones y niveles de pensamiento

En Lenguaje (Gráfica 3) se observa que la mayor fortaleza de los estudiantes (36\%) está en la dimensión del Hacer que según la Tabla de Especificaciones significa un adecuado manejo de estrategias sobre lectura y escritura, con desarrollo de la ortografía en la producción de diferentes tipos de textos. No obstante en esta misma dimensión un $43 \%$ de estudiantes alcanza un nivel Regularmente satisfactorio.

En la dimensión de Conocer, pocos estudiantes (17\%) manifiestan un óptimo manejo de conceptos sobre el aprendizaje de la lectura y escritura, sus dificultades y en la comprensión y producción de textos, demostrando un 54\% logro Regularmente satisfactorio.

De más a menos se observan los procesos de pensamiento en los estudiantes, demostrando una mayor movilización de pensamiento Deductivo (36\%), respecto de Crítico (25\%) y del Aplicado (20\%). En estos dos últimos casi el $60 \%$ de estudiantes logra un nivel Regularmente satisfactorio. 


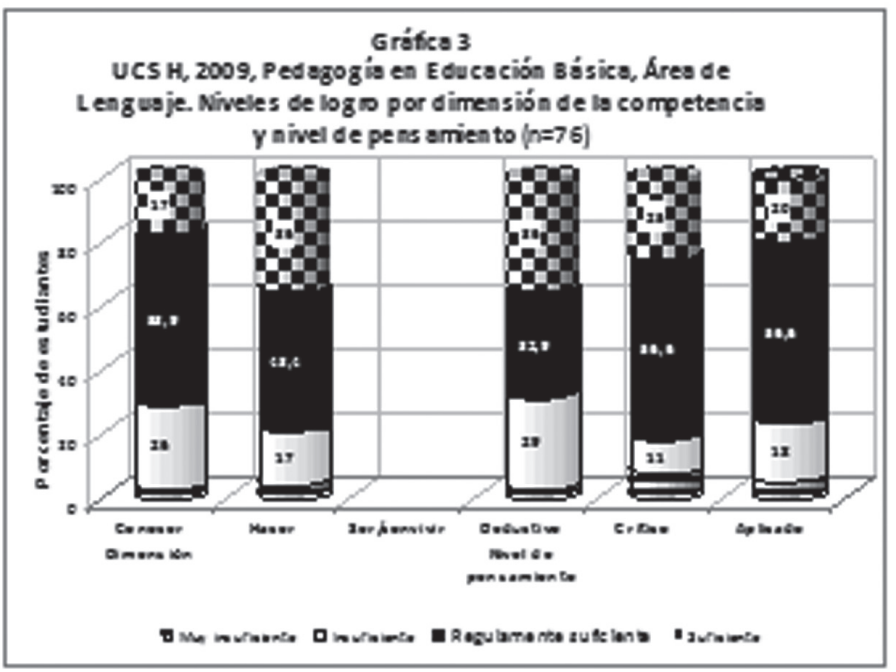

En suma, en Lenguaje, al relacionar las dimensiones de Conocer para saber Hacer, pareciera que los estudiantes que tienden a ser hábiles en el manejo de herramientas prácticas, aunque con relativa fundamentación disciplinar, por lo mismo, es posible que esto se refleje en la similitud de proporciones de estudiantes en aplicar el pensamiento Deductivo para saber Hacer, pero por otro lado, pueden ser señales de debilidades en la formación disciplinar que les proporciona el Conocer para saber Aplicar, siendo una base formativa importante para generar aprendizajes.

En Matemática (Gráfica 4) la dimensión del Hacer alcanza un logro Suficiente en un I5\% de los estudiantes del Programa evaluado y alude a la habilidades en procedimientos didácticos, operatoria, resolución de problemas, simbolismos y representaciones geométricas. Un 28\% lo logra en forma Regularmente suficiente. 


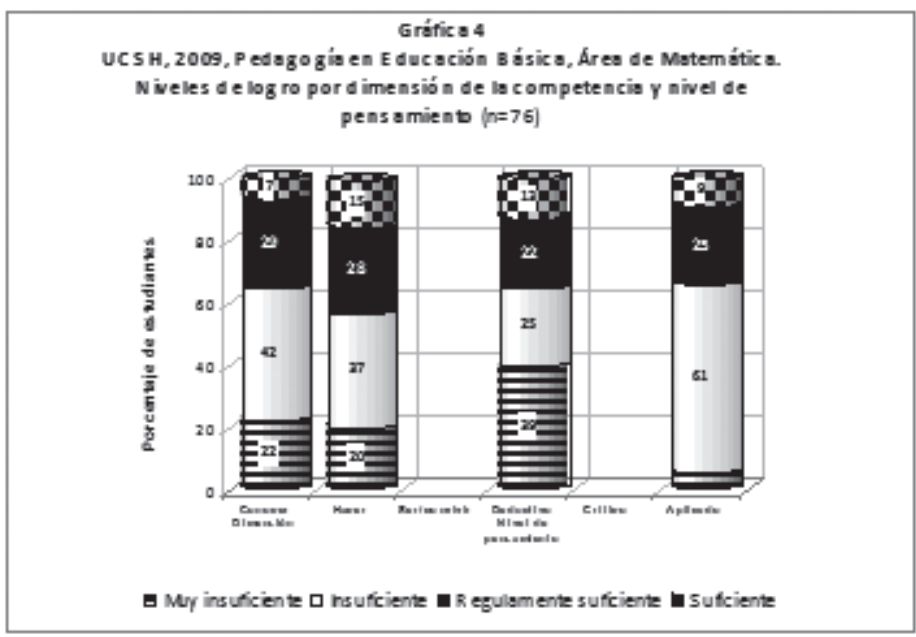

Un I3\% de estudiantes aplica pensamiento Deductivo en forma Suficiente y Aplicado en un $9 \%$ en contraposición con más del $60 \%$ en situación Insuficiente. En general, alrededor de un 20\% de la población estudiantil muestra logro Regularmente Suficiente en las dimensiones evaluadas con logros Muy insuficientes, altos especialmente en pensamiento deductivo.

En esta área, aunque con menos proporciones de estudiantes con logros Suficientes que Lenguaje, se observa que entre la dimensión del Hacer y el pensamiento Deductivo los porcentajes de estudiantes son parecidos, pudiendo entenderse como capacidad de los evaluados para desarrollar la didáctica de la enseñanza de la matemática a diversas situaciones, pero con limitaciones para transportar elementos teóricos disciplinares que faciliten la resolución de problemas, vistos también desde el saber Conocer para saber Aplicar.

\section{Planes comunes ${ }^{2}$}

Los resultados de este acápite son producto de la prueba institucional que aplicaron los estudiantes de PEB. No se hace distinción entre estudiantes por modalidad de jornada.

2 El PEB también fue analizado en el contexto de los demás programas de pedagogía de la UCSH, información disponible en el informe de Barrera, S., 2010a. 
Niveles de logros

En el PCU un tercio de estudiantes de PEB alcanza el nivel Regularmente suficiente, el resto se concentra en logro Insuficiente (65\%), comportamiento que se expresa en similares proporciones en los diferentes Ejes que lo componen, observándose en éstos pequeñas proporciones de estudiantes con fortalezas expresadas como logros óptimos deseables (Gráfica 5).

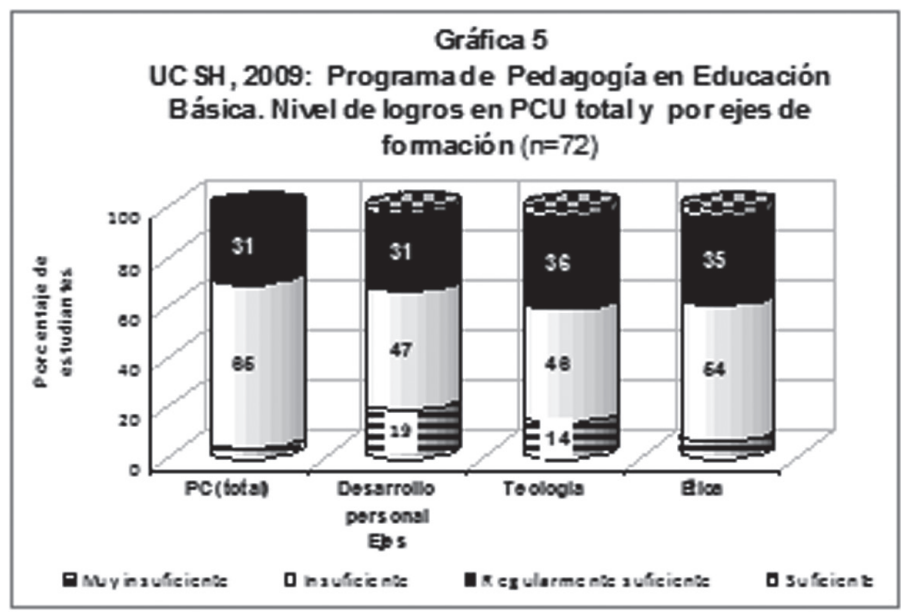

\section{Logros por Ejes del PCU}

Para analizar las Tablas I y 2 se adoptan los criterios de fortalezas y debilidades de las competencias, agrupadas dentro del Eje correspondiente. En ese marco comprensivo cobra sentido el análisis del Eje destacándose algunas proporciones de estudiantes. Considérese que en cada caso el número de ítems de la prueba institucional, en su segunda aplicación consecutiva, es suficiente para generar información válida y confiable.

Analizando en mayor detalle el logro de las competencias del PCU, en general se observan tres de mayor fortaleza, con fluctuaciones de I $9 \%$ y $24 \%$ de estudiantes con logro Suficiente. Se destacan:

- la competencia (9) de Desarrollo Personal (24\%) referida a la función de agente de cambio del docente, 
- la (2) de Teología (2I\%) sobre la importancia de la trascendencia de las personas, $y$

- la (4) de Ética (19\%) orientada a decisiones que comprometen el discernimiento en diferentes planos de actuación de las personas.

En el nivel de logro Regularmente satisfactorio se destacan dos competencias logradas por la mitad de los estudiantes, la (I) de Desarrollo Personal (50\%) que alude a la construcción de la autoimagen y el autoconcepto; la (4) de Ética (49\%) sobre la formación de criterios sobre el discernimiento ético en diferentes planos de aplicación.

Las competencias que agrupan a proporciones importantes de evaluados con logro Muy insuficiente son: la (6) de Ética (40\%) sobre la formación de criterios para la toma de decisiones, la (I) que potencia la autoimagen y el autoconcepto de las personas, y la (2) de Teología que compromete el sentido de trascendencia de la existencia humana (47\%), nótese que esta última tuvo un 21\% de estudiantes con logros óptimos.

Tabla 1

UC SH, 2009, Programa de Pedagogia en Educación Básica, nivel de logro de las competencias UCSH, 2009, Programa de Pedagogia en Educación Básica
PCU pojes

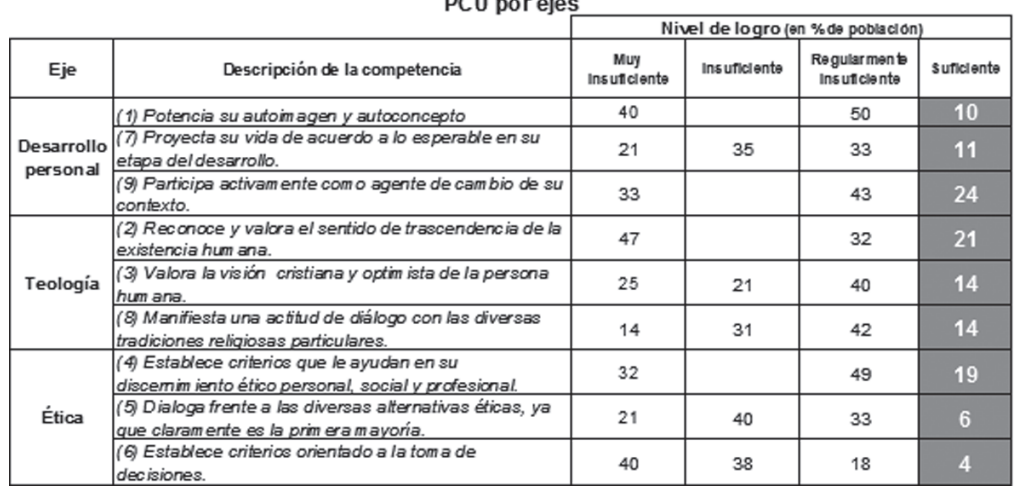

El PCEDUC (Gráfica 6), en un análisis de contraste de resultados de conjunto, se observa relativamente mejor logrado que el PCU, porque muestra alrededor de un 60\% de estudiantes con logros Regularmente suficientes en sus Ejes, y en todos los Ejes se evidencian repuntes de proporciones de estudiantes con logros Suficientes. 
Por desagregación de Eje, Planificación comporta una mayor fortaleza con logro Satisfactorio (I4\%), los otros Ejes muestran proporciones más modestas en este mismo nivel de logro. Clima de aula (67\%) es mayor logro relativo, seguido de Gestión de la enseñanza (6I\%).

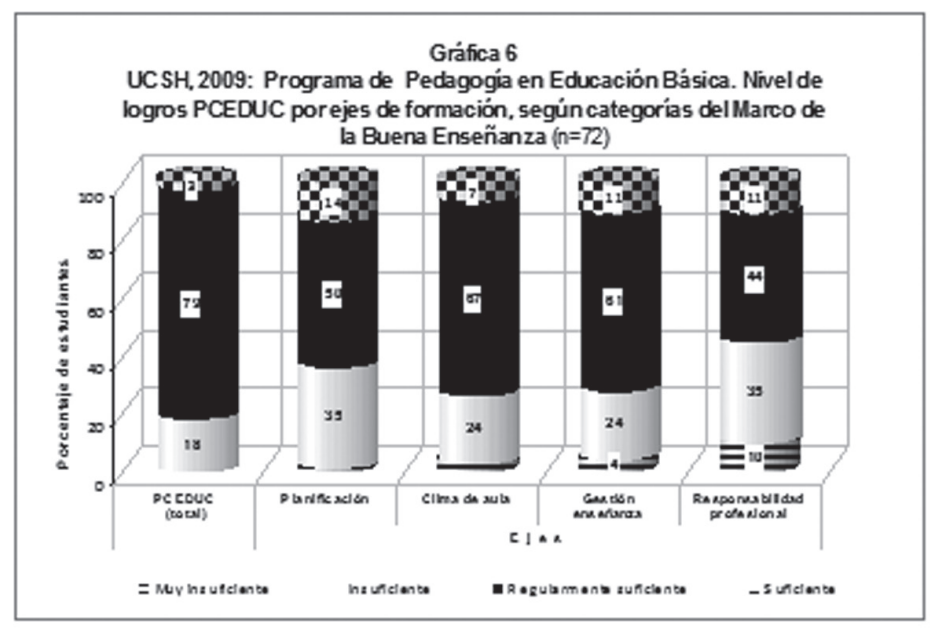

Las competencias con mayor fortaleza por Ejes del PCEDUC expresadas en logro Suficiente son (Tabla 2):

- la competencia 8 del Eje de Gestión del aprendizaje sobre el desarrollo de habilidades de comunicación con los estudiantes (33\%),

- luego en Clima de aula, la 5 sobre capacidad para generar dentro de la clase espacios propicios para el aprendizaje (26\%),

- y en Planificación de la enseñanza la I con un 21\% de evaluados que demuestra habilidades para integrar las características de los estudiantes, sus conocimientos y experiencias previas a los objetivos de aprendizaje y la 3 que comporta un adecuada selección de estrategias y recursos acorde a las características de diversidad de los estudiantes (19\%).

Con logros Regularmente satisfactorios se observan tres competencias del PCEDUC que se enfocan en altas proporciones de evaluados; la I y la 3, ambas de Planificación de la enseñanza, a la primera ya se hizo referencia y cuenta con $63 \%$ de estudiantes en este nivel, y la segunda 
comporta la habilidad de los evaluados (56\%) en seleccionar recursos metodológicos, ajustada al desarrollo y condiciones de diversidad de los estudiantes.

En Gestión del aprendizaje (9) se concentra también un 56\% de estudiantes hábiles en aplicar estrategias didácticas adecuadas, le sigue la 6, de Clima de aula (47\%) que alude a la capacidad de los evaluados en comunicar sus expectativas de aprendizaje a los escolares.

Con estas evidencias dadas por los datos presentados, de manera general se puede puntualizar que, aunque en los dos Planes Comunes se observan bajas proporciones de estudiantes con logros óptimos, en PCEDUC se observa un repunte relativo comparativamente mayor, además cuatro de ocho competencias evaluadas se destacan con logros suficientes, mientras que en PCU ocurre algo similar con tres de las nueve competencias. Otra diferencia es que el PCEDUC tiene más nutrido el nivel de logro Regularmente suficiente, mientras que PCU el logro Muy insuficiente.

La mayor fortaleza de los estudiantes de PEB en PCU está en dos competencias, una que propugna el involucramiento y compromiso docente en procesos de cambio y el desarrollo del sentido de trascendencia de las personas, y en PCEDUC son varias: el desarrollo de habilidades comunicacionales con los escolares, la creación de un adecuado clima de aula que predispone al aprendizaje y la consideración de las características de los escolares para hacer clases efectivas.

A contrapunto de los resultados de la prueba generalista, tómese en cuenta de todas maneras, las insuficiencias o debilidades disciplinares observadas en las dimensiones de las competencias, especialmente respecto de las limitaciones en conocimiento disciplinar y transposición didáctica correspondiente. 


\section{DIFICULTADES EN LA PRÁCTICA PROFESIONAL ${ }^{3}$}

De los 253 casos consignados en la evaluación de los 10 programas de pedagogía de la UCSH, se filtraron los correspondientes al Programa de PEB, cuya información fue sistematizada a efecto de incluirla en el presente Informe.

En este acápite se presenta y analizan primero, de manera global, dos gráficas sobre los practicantes del Programa de PEB, con las frecuencias de distribución de casos, según:

- las categorías de dificultades encontradas en la práctica, y

- la identificación de los actores involucrados.

Se desechó la generación de una tabla de contingencia porque los datos son pocos y salen muy dispersos en la distribución, siendo más provechosa la explotación de la información cualitativa por las categorías de dificultades levantadas desde los testimonios, y analizándolos en su integridad, teniendo en cuenta intrínsecamente al tipo de sujeto al que el evaluado alude y que pudiera tratarse de más de uno, por ejemplo, una dificultad en la enseñanza de la matemática desde el practicante, también involucra al estudiante que no gusta de la asignatura, en casos similares predomina el problema y el sujeto activo.

\section{Levantamiento de categorías de dificultades}

La Gráfica 7 representa las 10 categorías construidas en el análisis de la experiencia realizada en 2009 con los Programas de Pedagogía de la UCSH. En la oportunidad emergieron ocho categorías; en esta segunda experiencia se identifican dos más relacionadas con Necesidades Especiales y Contexto y riesgo social, todas ilustradas con expresiones de los propios evaluados. El Programa de PEB no estuvo exento, prueba de ello son los testimonios de respaldo que se ejemplifican en el informe técnico in extenso existente.

3 Op. Cit. pp. 42 a 49 y Anexo 9, pp. I27-I28, información complementaria disponible sobre categorías, descriptores y testimonios de los practicantes. 
De mayor a menor se observan las frecuencias de casos categorizados por temática de dificultad. Entre todas las categorías, sobresale la frecuencia de casos y recurrencia con que se alude al Acompañamiento entendido como el diálogo continuo entre el profesor Supervisor de la universidad y el profesor de curso, denominado Colaborador, para orientar en la reflexión del proceso y en la resolución de problemas que se le presentan al practicante en el aula.

Le siguen en frecuencia las dificultades asociadas a la Enseñanza entendida como las acciones y estrategias programadas que realiza el practicante en la clase, con aplicación de didácticas específicas y recursos facilitadores del aprendizaje de los estudiantes.

\section{Disciplina, Aprendizaje y Gestión escolar}

En tercer orden los practicantes consultados se refieren a situaciones de Disciplina y Convivencia que se les enfrentan en su trabajo representado por situaciones conflictivas con expresiones de agresividad de sus estudiantes, interrupción de la clase, y pérdida de autoridad. Al mismo nivel de incidencia, los estudiantes destacan problemas de Aprendizaje dentro del cual identifican desmotivación, desatención, en clases y evidencias de bajo logro en los escolares. En este mismo bloque de frecuencias se relevan problemas en la Gestión en la Unidad Educacional (UE) que comportan relaciones de comunicación, organización y coordinación de acciones y uso de recursos de apoyo a la labor del practicante y que limitan la posibilidad de desarrollar un trabajo más eficiente.

Planificación, Formación y Acogida/oganización de la práctica

Con frecuencias menores, en la misma gráfica se registran casos que refieren a dificultades en manejo de Instrumentos y planificación que incluye manejo de jefatura de curso, libro de clases y administración del tiempo de clase. Los problemas relacionados con la Formación profesional que alude a la aplicación de la teoría aprendida, y 
cómo llevar la construcción del aprendizaje en el estudiante, que guarda sentido con la necesidad de profundizar en didácticas y conocer herramientas de gestión académica. Esta categoría está muy relacionada con la naturaleza de problemas que se le presentan al practicante en orden a la Organización de la práctica y acogida en las UEs que escapa a su control porque son de responsabilidad institucional ya sea de la escuela o de la universidad que les asigna la unidad de práctica; tal es el caso de la distancia, costos de transporte, remuneración de la práctica y el control de sobretiempos, por identificar algunos.

\section{Necesidades especiales y Contexto}

Finalmente, no por tener menos frecuencia dejan de ser importantes problemas relacionados con la atención de escolares con Necesidades especiales, sobre todo cuando se trata de casos que requieren de ayuda especializada frente a los cuales tampoco la UE toma acción.

Los problemas relacionados con el Contexto y riesgo social de evidente raíz estructural fueron caracterizados por los practicantes como espacios escolares donde ineludiblemente se ven interpelados por problemas de drogadicción y delincuencia, causándoles presiones emocionales difíciles de manejar, considerando no estar preparados para enfrentarlas.

Tampoco los practicantes creen que sea la universidad la que deba formarles para enfrentar estas experiencias, no obstante, la compleja realidad escolar también es expresión de la compleja sociedad, y en ella se expresan sus problemas, frente a los cuales a las instituciones formadoras de profesores les corresponde pronunciarse sobre sus límitaciones y alcances. 


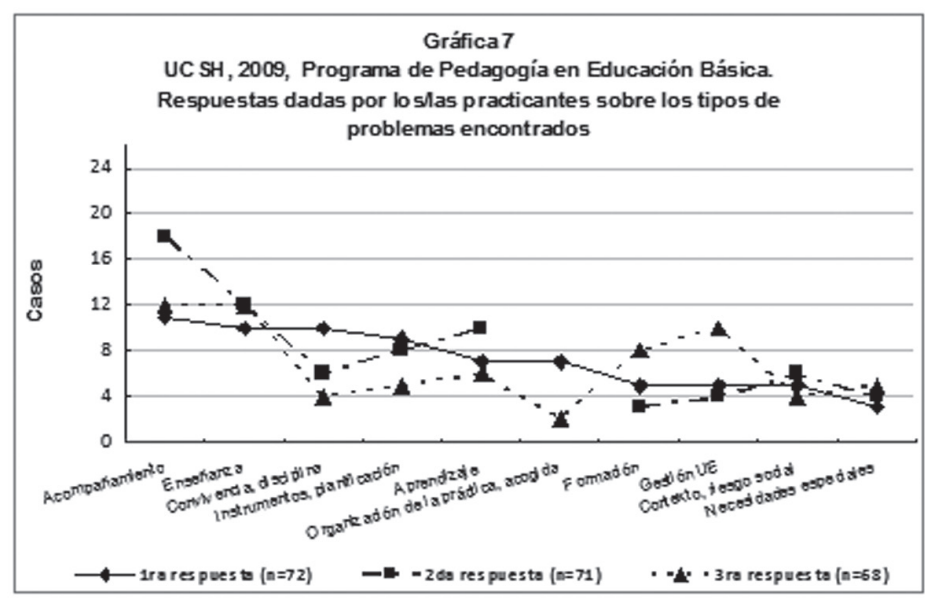

Actores sociales involucrados

La Gráfica 8 identifica en número de casos evaluados a ocho actores vinculados directa o indirectamente a los tipos de problemas identificados en el punto anterior.

Los datos están ordenados de mayor a menor frecuencia. La lra respuesta de los practicantes tiene un comportamiento decreciente de casos que aluden a diferentes actores. Se observan dos que sobresalen como protagonistas en los diferentes tipos de problemas encontrados en el punto anterior, coincidencialmente con el mismo número de frecuencias (24 casos del total): el propio Practicante y los Profesores Guía y Supervisor. En otras palabras, las dificultades que enfrentan los practicantes como enseñantes noveles y el limitado apoyo que Guías y Supervisores les proporcionan.

Le sigue el Estudiante o escolar del curso encomendado, luego la Unidad educacional como institución de acogida a los practicantes.

Los Profesores de la UE, que son referentes importantes del contexto social en que los practicantes son considerados también actores, toda vez que los practicantes se integran a una estructura orgánica y un cuerpo social institucional, actores asociados probablemente a las dificultades sobre acogida de los practicantes en el ambiente de trabajo. 
La mención que hacen los practicantes a la Familia está matizada de testimonios de los practicantes en los que expresan su percepción sobre la despreocupación por sus hijos, especialmente de aquellos con dificultades en el aprendizaje.

La identificación de la Universidad como actor implicado en algún tipo de dificultades encontradas por los practicantes, representan I4 casos de los 72 evaluados de PEB. Si bien son pocos, es preciso analizar con detenimiento los testimonios que argumentan problemas de la categoría Formación y Acompañamiento, y en algunos casos Organización de la práctica, porque se está ante variables dinámicas que pueden ser modificadas favorablemente mediante la toma de decisiones de orden académico, curricular u organizativo institucional, con reorientación de acciones, énfasis o inclusión de elementos formativos no profundizados o emergentes, a diferencia de otros problemas difíciles de intervenir por su naturaleza estructural o sistémica.

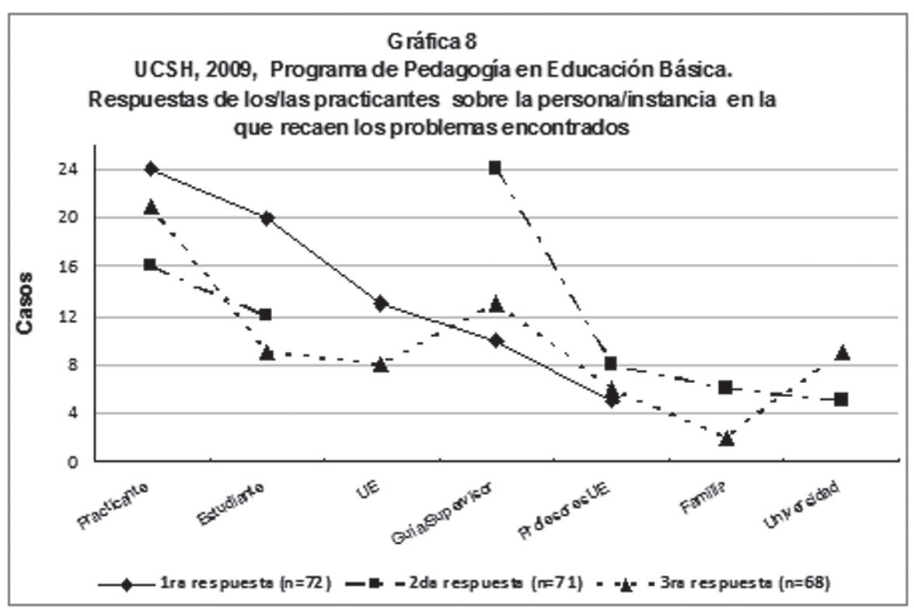

\section{Reflexión final}

La responsabilidad compartida del Estado y las instituciones de educación superior formadoras de capital humano se expresa en debates abiertos sobre temas de interés y sensibilidad pública que posteriormente se refrendan en leyes que se vuelven políticas públicas. Siendo así, la calidad de los procesos formativos y de los profesionales 
que egresan y se insertan en la sociedad son un compromiso que va más allá de la voluntariedad y las intenciones.

La calidad es un concepto presente en todos los planos de la vida y conlleva conjuntos de atributos que tienen un valor para quienes los determinan, además de significados y connotaciones diversas, dependiendo de tradiciones históricas y culturales de las personas. Refleja una forma de concebir el mundo, del ser, hacer, estar y desear; la educación es una de ellas, donde el ser humano es el sujeto y el objeto. Pero la educación también es ideología, intereses, tensiones y contradicciones; es ética, es bien común y felicidad, como dirían Platón y Aristóteles.

Con el desarrollo de la ciencia, y el progreso del hombre y su desarrollo, la rendición de cuentas en los actuales tiempos surge como una necesidad de responder por los actos públicos y la consecución de mandatos, y dentro de éstos las personas y las instituciones están llamadas a evaluarse para estimar el grado de cumplimiento de la calidad.

Las prácticas evaluativas en el sistema educacional chileno llevan casi medio siglo y continúan instalándose con diversos propósitos, por un lado, en aras de mejorar la calidad de los procesos y sus resultados, expresados en mejores aprendizajes, y por otro, para seleccionar a la población escolar para la continuidad de estudios superiores o dar incentivos.

Desde la institucionalización de la otrora Prueba de Aptitud Académica hasta hoy, el propósito central no ha cambiado sustancialmente. La tendencia parece alejar a la evaluación de su bondad valorativa y constructiva, bajo escrutinio reflexivo, comprensivo para el aprendizaje de una realidad e iluminar el camino de la mejora continua, o de la transformación y el cambio educacional. Las señales de los tiempos acercan a la evaluación cada vez más al inveterado despropósito castigador, sancionador, culposo e inquisidor. ¿Estamos avanzando o retrocediendo? ¿Qué desencadena cuando su uso es trastrocado? ¿De quién depende que así sea? ¿Seguiremos en la misma pendiente? 
¿A dónde conducen los abusos y excesos de la evaluación? ¿Estamos al servicio de la evaluación o la evaluación está a nuestro servicio?

Evaluar a los egresados de pedagogía comenzando por la carrera de PEB es bueno porque se va casi a la raíz socializadora formal, es pertinente acompañar los procesos formativos para saber donde están sus debilidades y fortalezas, mejorar unas y potenciar otras. No obstante, es preciso comunicar, difundir, transparentar y rendir cuentas también quienes llevan a cabo la investigación y la gestión evaluativa, para conocer la existencia de artilugios del oficio o evidenciar la cientificidad con que se llevan a cabo.

La presente experiencia se suma a la espiral que tienen el foco en estudiantes e instituciones en diferentes niveles del sistema: escolares, egresados de media, unidades educacionales, docentes del sistema municipal y subvencionado, instituciones de educación superior técnica y universitaria y egresados de carreras de medicina, pedagogía básica y educación parvularia.

Se parte de la base de que el compromiso con la calidad educacional tiene como "amigo crítico" a la evaluación, por lo tanto, lejos de rechazarla, su democratización comporta la participación de los involucrados y en ese camino se crean condiciones subjetivas de apropiación, y socialización que predisponen al cultivo de la evaluación. Si a esto le agregamos una forma de evaluar combinando enfoques cuantitativos y cualitativos, la posibilidad de comprensión del objeto, variables, dimensiones, o factores que pudieran estar incidiendo en los resultados se acrecientan y profundizan.

Bajo estas consideraciones la experiencia piloto de evaluación del perfil de egreso de los estudiantes en la UCSH abre en la institucionalidad un surco fecundo para el cultivo de la evaluación, y acercar a los actores a pensar en la calidad de los procesos protagonizados, el rigor, los niveles de exigencias propuestos, el alcance y concreción de las competencias declaradas en los programas curriculares y en el Modelo de Formación. 
Más allá de aceptar o rechazar los resultados, la actitud de apertura de los diferentes usuarios al análisis y la reflexión crítica de los procesos formativos desarrollados, abona el camino a la mejora permanente, donde ahora el docente formador pasa a ser otro eje susceptible de ser evaluado en su desempeño en relación a los logros de sus estudiantes, no como algo separado, lineal, con finalidad de cumplido administrativo.

Que los resultados evidencien que lenguaje está relativamente mejor logrado que matemática, o que ciencias naturales concentre logros regularmente logrados, o que ciencias sociales y educación artística muestren insuficiencias, debe motivar la revisión de las competencias definidas, procesos pedagógicos, el énfasis o no en la formación disciplinar o la subordinación de cuestiones técnicas e instrumentales emergentes por parte del sistema educacional, como también la dinámica curricular formativa e introducir ajustes para hacerlos pertinentes a las demandas del entorno social nacional e internacional.

Por otra parte, aunque algunas competencias de orden genérico referidas al PCEDUC, transversal a los programas de pedagogías, en el caso de PEB tienen buenos logros, especialmente en procesos comunicacionales en referencia a los escolares, así como también la creación de clima de aula, la función del docente como agente de cambio en su contexto, junto con el sentido de trascendencia de la persona (PCU), es preocupante la preponderancia de egresados con logros insuficientes en ambos Planes, especialmente en PCU. La universidad propicia el desarrollo personal, social y ético de sus estudiantes bajo el denominado sello identitario salesiano. ¿En qué medida éste se da en la profundidad debida como para que los egresados al momento de responder ítems de resolución de problemas, orienten sus respuestas dentro de la postura deseable? Cumplidas las formalidades curriculares, ¿cómo están dispuestas en la malla curricular las competencias del sello universitario para que pemeen en toda la formación? 
La información sobre dificultades sorteadas por los egresados durante el ejercicio de la práctica profesional y sus opiniones sobre el proceso formativo recibido en la UCSH, son un complemento comprensivo importante a la luz de las competencias formativas evaluadas. Las categorías levantadas a partir de los testimonios de los evaluados, pueden tener una lectura interpretativa con las dimensiones matriciales consideradas en las tablas de especificaciones tanto de pensamiento como del saber, pudiendo el egresado desarrollar procedimientos hasta donde los aprendizajes disciplinares se lo permitan.

Algunas propuestas que pueden ser consideradas por los usuarios de la evaluación son:

- La necesidad de concebir un sistema integrado de indicadores, y poner en marcha un sistema de vigilancia, prevención y aseguramiento de la calidad de la formación profesional que engrane con las prácticas evaluativas de los programas.

- La importancia de gestionar indicadores integrados por cohorte de estudiantes para elaborar series y tendencias, para identificar oportunamente estudiantes con rezago y brindarles el soporte tutorial necesario.

- La articulación entre procesos evaluativos de aula y el logro de competencias formativas por actividad curricular, con la introducción de técnicas de valoración cualitativa como las Rúbricas y el acompañamiento respecto al estudiante de bajo logro. De este modo se facilita en los académicos la comprensión del manejo de los niveles de logros que se diferencia del rendimiento académico traducido en notas, aun cuando en últimas el nivel de logros se reconvierta en una escala de notas, a efectos administrativos. Esta práctica evaluativa permite al docente, con conocimiento de causa, hacer realidad el acompañamiento a estudiantes de bajo desarrollo de las competencias. La responsabilidad por los resultados es parte de la cultura evaluativa.

- Continuar con evaluaciones institucionales anuales del logro de los perfiles de egreso, mejor si se decide incluir también los perfiles intermedios comandados por las instancias de gestión 
de los propios programas. Complementar líneas temáticas de investigaciones en metodología y didáctica, así como también sobre los estudiantes y variables asociadas a su capital cultural y social. - Desde la universidad generar opinión pública sobre la educación como servicio social, y la toma de posición de sus egresados frente a la atención educacional, contextos vulnerables para incidir en esa línea, a fin de proporcionarles herramientas susceptibles de ser empleadas en el manejo de la conflictividad escolar y desarrollar estrategias pedagógicas y de clima dentro del aula a favor de la calidad y calidez del ambiente propicio para aprender.

Finalmente, se destaca la importancia de la participación propositiva de la UCSH en espacios técnicos convocados por el Mineduc, ya que contribuye al mayor consenso hacia e el establecimiento de un currículo común para las universidades que forman profesores (aun cuando cada una de ellas introduzca particularidades, énfasis o competencias identitarias). Siendo así, las prácticas evaluativas pueden ser reorientadas en base a parámetros de referencia común, pero sin comparar resultados entre universidades.

\section{Bibliografía}

Abadzi, H. (2008). Aprendizaje Eficaz y Pobreza. Orientaciones para el desarrollo. Ideas desde la frontera de la neurociencia cognitiva. Santiago: Ediciones UCSH.

Araya-Ramírez, C.; Taut, S.; Santelices, V.; Manzi, J. \& Miño, F. Teoría subyacente del Programa de Asignación de Excelencia Pedagógica en Chile. España: Revista de Educación, 359. Septiembre-diciembre 2012 (en prensa). Pontificia Universidad Católica de Chile. Facultad de Ciencias Sociales. Escuela de Psicología. Centro de Medición. Santiago de Chile. Disponible en: http://www.revistaeducacion.mec.es/doi/359_I06.pdf

Ávalos, Beatrice (2002). Profesores para Chile. Historia de un Proyecto. Santiago: Ministerio de Educación. Colorama.

Barber, M. \& Mourshed, M. (2008). Cómo hicieron los sistemas educativos con mejor desempeño del mundo para alcanzar sus objetivos. Perú: PREAL No 4I. Disponible en: http://www.educacion.gob.es/cesces/estudios/ informe-mckinsey-2008.pdf 
Evaluación del perfil de egreso y prácticas profesionales en estudiantes de Pedagogía en Educación Básica, en la Universidad Católica Silva Henríquez (UCSH), 2009 - Susana Barrera A.

Barrera, S. (2009). Informe técnico. Una experiencia piloto en la UCSH: Evaluación de competencias de los Planes comunes de universidad y de educación, en egresados de Programas de Pedagogía, gestión 2008. Santiago de Chile: Dirección de Docencia de Pregrado, UCSH.

Barrera, S. (2010a). Informe técnico. Evaluación de perfiles de egreso en estudiantes de Programas de Pedagogía, gestión 2009, Planes comunes de universidad y de educación. Santiago de Chile: Dirección de Docencia de Pregrado, UCSH.

Barrera, S. (2010b). Informe técnico. Experiencia piloto Evaluación del perfil de egreso, Programa de Pedagogía en Educación Básica, gestión 2009. Santiago de Chile: Dirección de Docencia de Pregrado, UCSH.

Biblioteca del Congreso Nacional. Ley 20.129 (17/ll /2006) de Aseguramiento de la Calidad de la Educación Superior. Disponible en: http:/ / www.leychile. $\mathrm{cl} /$ Consulta/listaresultadosimple?cadena=Aseguramiento $\% 20 \mathrm{de} \% 20$ la\%20calidad

Biblioteca del Congreso Nacional. Ley 19.175 (3I/0I/200I). Otorga un mejoramiento especial de remuneraciones para los profesionales de la educación. Disponible en: http://www.leychile.cl/Consulta/listaresultadosimple ?cadena $=19715$

Centro Interuniversitario de Desarrollo (2004). Competencias de egresados universitarios. Santiago de Chile: Alfabeta Artes Gráficas.

Consejo de Rectores, Universidades Chilenas (2008). Estudio acerca de la validez predictiva de los Factores de Selección a las Universidades del Consejo de Rectores. Comité Asesor. Documento Técnico, Santiago de Chile: CRUCH.

Consejo Superior de Educación (2010). Calidad de los egresados, responsabilidad institucional ineludible. Seminario Internacional. Santiago de Chile: Impreso en Andros Impresores.

Contreras, M.; Corvalán, F. \& Redondo, J. (2007). Cuando la suerte está echada. Estudio cuantitativo de los factores asociados al rendimiento de la PSU. Santiago de Chile: Universidad de Chile, Facultad de Ciencias Sociales.

Darling-Hammond, L. \& McLaughlin, M.W. (2004). Políticas que apoyan el desarrollo profesional en una época de Reforma. Profesorado, revista de currículum y formación del profesorado 8 (2). Universidad de Stanford. Disponible en http://www.ugr.es/ recfpro/rev82COLI.pdf

Darling-Hammond, Linda (2000). Teacher Quality and Student Acbievement: A Review of State Policy Evidence. Educational Policy Analysis Archives. Stanford University. Vol. $8 \mathrm{~N}^{\circ}$ I. Disponible en: http://epaa.asu.edu/epaa/ v8nI/

Larrondo, T. (200I). Pruebas de babilidades básicas. Presentación de datos. 
Larrondo, T. (2007). Desarrollo de babilidades básicas en lenguaje y matemática en egresados de pedagogía. Un estudio comparativo. Santiago: Consejo Superior de Educación. Disponible en www.cse.cl

Millman, J. \& Darlimg-Hammond, L. (Editores) (I997). Manual para la evaluación del profesorado. Madrid: La Muralla.

Ministerio de Educación, CPEIP (2009). Presentación de Resultados. Evaluación Diagnóstica 2008. Santiago de Chile. 2009.

Ministerio de Educación (2009). SIMCE. Unidad de Currículum y Evaluación. Resultados SIMCE 2008, 4to educación básica y 2do educación media. Santiago de Chile, mayo 2009. Disponible en: http://www.simce.cl/

Ministerio de Educación (2008a). Entrega de resultados SIMCE 2007. Intervención Ministra. Biblioteca Nacional. Santiago. Disponible en: http://www.mineduc.cl/biblio/documento/

Ministerio de Educación (2008b). Estrategia Bicentenario para la Formación Docente.

Ministerio de Educación CPEIP (2003). Marco para la Buena Enseñanza. Santiago: C\&C Editores.

Ministerio de Educación, CPEIP. Programa de Asignación de Excelencia Pedagógica. Disponible en http://www.aep.mineduc.cl/legal.asp

National Board for Professional Teaching Standard (NBPST). Teacher Preparation E Professional Development. Disponible en: http://www.nbpts.org/ policy_center/education_policy_higher

OCDE (20II). Mejores políticas para el desarrollo: Perspectivas OCDE sobre Chile, OECD Publishing. Disponible en: http://dx.doi. org/IO.I787/9789264095755-es

Rodríguez, Gregorio; Gil, Javier y García-Jiménez, Eduardo (1996). Metodología de la Investigación cualitativa. Imprime T.G. Arte, Jubería y Cía. S. L. Granada, España.

Schön, Donald (2002). La formación de profesionales reflexivos. Barcelona: Paidós. Ministerio de Educación y Ciencia.

UCSH (2003). Modelo de Formación de la Universidad Católica Silva Henríquez, Santiago de Chile: UCSH.

UCSH (2006). Propuesta de Plan Común de Educación. Documento de trabajo. Santiago de Chile: Departamento de Educación. Coordinación académica.

UCSH (2008). Información Institucional de Evaluación. Santiago de Chile: UCSH. 
Evaluación del perfil de egreso y prácticas profesionales en estudiantes de Pedagogía en Educación Básica, en la Universidad Católica Silva Henríquez (UCSH), 2009 - Susana Barrera A.

UNESCO (2000). Primer estudio internacional comparativo sobre lenguaje, matemática y factores asociados para alumnos del tercer y cuarto grado de la educación básica. Segundo Informe. Laboratorio Latinoamericano de Evaluación de la Calidad de la Educación. Disponible en: http://unesdoc.unesco.org/ images/0012/00123I/I23I43s.pdf

UNESCO (2008). Los aprendizajes de los estudiantes en América Latina y el Caribe. Segundo estudio regional, comparativo y explicativo. Laboratorio Latinoamericano de Evaluación de la Calidad de la Educación. Santiago de Chile: Impresores Salesianos. 